\title{
Low-dose fractionated radiation with induction chemotherapy for locally advanced head and neck cancer: 5 year results of a prospective phase II trial
}

\author{
John F. Gleason Jr. • Mahesh Kudrimoti • \\ Emily M. Van Meter • Mohammed Mohiuddin • \\ William F. Regine • Joseph Valentino • Daniel Kenady • \\ Susanne M. Arnold
}

Received: 5 September 2012 / Accepted: 10 October 2012 /Published online: 20 October 2012

(C) Springer-Verlag Berlin Heidelberg 2012

\begin{abstract}
Objective This study aims to report the long-term outcomes of a novel treatment approach utilizing induction low-dose fractionated radiation therapy (LDFRT) and chemotherapy for locally advanced squamous cell carcinoma of head and neck (SCCHN). Methods We prospectively enrolled 40 patients with locally advanced SCCHN (77\% stage IV) on a phase II clinical trial
\end{abstract}

This work was presented in abstract and poster form at the 53rd Annual Meeting of the American Society for Radiation Oncology (ASTRO), Miami, FL, October 2nd-October 4th, 2011.

Supported by an unrestricted research grant from Bristol-Myers Squibb. The Lexington Veterans Administration Medical Center provided resources and the use of facilities to support this research investigation.

J. F. Gleason Jr. • M. Kudrimoti

Department of Radiation Medicine,

University of Kentucky, Markey Cancer Center,

Lexington, KY, USA

E. M. Van Meter

Division of Cancer Biostatistics,

University of Kentucky, Markey Cancer Center,

Lexington, KY, USA

M. Mohiuddin

King Faisal Specialist Hospital and

Research Centre,

Riyadh, Saudi Arabia

W. F. Regine

Department of Radiation Oncology,

University of Maryland,

Baltimore, MD, USA

J. Valentino

Department of Otolaryngology HNS,

University of Kentucky, Markey Cancer Center,

Lexington, KY, USA and treated with induction paclitaxel $(225 \mathrm{mg} / \mathrm{m} 2)$, carboplatin (AUC 6), and LDFRT ( 80 cGy BID on days 1 and 2) every 21 days for two cycles.

Results Forty patients enrolled; 39 were evaluable. The acute toxicity and response data have been previously reported; overall response rate (RR) was $82 \%$. After induction, definitive therapy was concurrent chemoradiation (CRT) in $51 \%$,

J. Valentino

Department of Surgery, University of Kentucky,

Markey Cancer Center,

Lexington, KY, USA

D. Kenady

Division of General Surgery, Department of Surgery,

University of Kentucky, Markey Cancer Center,

Lexington, KY, USA

S. M. Arnold $(\bowtie)$

Division of Medical Oncology, Department of Internal Medicine,

Markey Cancer Center, cc445,

800 Rose Street,

Lexington, KY 40536, USA

e-mail: smarno0@uky.edu 
XRT alone in $39 \%$, surgery in $5 \%$, and surgery and XRT in $5 \%$. The long-term outcomes are now reported with a median follow-up of 83 months. Locoregional control (LRC) is $80 \%$ and distant control (DC) is $77 \%$. Five-year overall survival (OS), disease-specific survival, and progression-free survival (PFS) are $62 \%, 66 \%$, and $58 \%$, respectively.

Conclusion Induction chemotherapy with LDFRT has a high initial RR, comparable toxicity to two-drug induction regimens, but adds a third novel and effective agent, LDFRT. Five-year follow-up shows favorable outcomes compared to historical controls and excellent compliance with definitive therapy. This novel treatment approach is now planned for phase 3 trial evaluation.

Keywords Low-dose $\cdot$ Radiation $\cdot$ Induction Chemotherapy

\section{Introduction}

Over 50,000 patients will be diagnosed with squamous cell carcinoma of the head and neck (SCCHN) in the United States in 2011 [1]. Locally advanced disease (stages III, IVA, and IVB) makes up the majority of cases, and historical survival outcomes in these patients range from $30 \%$ to $40 \%$. Both local and distant recurrences are an issue in this patient population [2].

Systemic chemotherapy (most frequently platinumbased) has been added to locoregional therapies in an attempt to improve outcomes in these patients. Modern randomized trials have shown that the addition of concurrent chemotherapy to definitive radiation improves locoregional control (LRC), organ preservation, disease-free survival, and in some instances overall survival [3-6]. The addition of concurrent chemotherapy to post-operative radiotherapy has also shown to be beneficial in the high-risk subsets of patients with positive margins and extracapsular extension [7-9]. The results of these and other trials are further bolstered by a large meta-analysis, which at the most recent update, including over 17,000 patients, show a $4.5 \%$ overall survival benefit at 5 years with the addition of chemotherapy to locoregional treatment alone (radiation and/or surgery). The absolute benefit was largest $(6.5 \%)$ when the chemotherapy was administered concurrently. Induction chemotherapy also showed a modest improvement in this metaanalysis, $2.4 \%$ at 5 years [10].

Multiple evidence-based treatment approaches are now available for locally advanced SCCHN. These include surgery followed by radiation (or chemoradiation), definitive chemoradiation, altered fractionation radiation therapy alone, induction chemotherapy followed by chemoradiation or radiation alone, and radiation with concurrent EGFR inhibition. As locoregional control has begun to improve these advances, there has been a renewed interest in induction chemotherapy as a possible way to improve overall survival and decrease the high doses of radiation required currently for definitive management. Spurred by the MACH-NC meta-analysis [10] showing an overall survival benefit for induction chemotherapy, two recent trials have shown the superiority of taxanecontaining induction regimens: TAX 323 [11] and TAX 324 [12] both compared induction TPF (docetaxel, cisplatin, and 5-fluoruracil) versus PF but had different definitive therapies post-induction (radiation in TAX 323 and chemoradiation in TAX 324). TAX 323 demonstrated improvements in progression-free and overall survival at 5 years in the TPF arm [11], while TAX 324 showed an improvement in 5-year overall survival in the TPF arm $[12,13]$. The biggest drawback to the use of induction TPF is the significant acute toxicity ( $>75 \%$ grade 3 or 4 neutropenia in both trials) of the three-drug combination.

One alternative approach is to utilize low-dose radiation, which has low toxicity, with a two-drug induction regimen. In this way, radiation takes on the role of potentiating the chemotherapy without causing significant toxicity. Lowdose fractionated radiation therapy (LDFRT) $(<100 \mathrm{cGy})$ is a unique biologic phenomenon, characterized by Joiner et al. [14], who demonstrated that at the lowest doses, hyperradiosensitivity (HRS) exists [15]. Our institution has reported that LDFRT potentiates the effect of chemotherapy in vitro and in animal models; preclinical data indicate a range of 50 to $80 \mathrm{cGy}$ of low-dose radiation exploits this HRS phenomenon. In addition, four fractions of LDFRT demonstrated maximal cell death when combined with chemotherapy in vitro $[16,17]$. This discovery led to the design of the present trial combining the power of LDFRT with induction chemotherapy in locally advanced SCCHN. Induction chemotherapy allowed for rapid assessment of whether HRS was a translatable biologic phenomenon through RR and long-term survival assessment. The initial results of this unique treatment paradigm reported on the response rates and safety using an induction regimen of paclitaxel, carboplatin, and LDFRT [18], and we now present the long-term follow-up data.

\section{Methods}

Patient characteristics and eligibility criteria

This was a phase II prospective trial in stage III and IVa/b SCCHN. From July 2000 to May 2002, 40 adult patients were consented per institutional and federal guidelines to receive LDFRT, paclitaxel, and carboplatin (UK H\&N-11). Eligible patients had pathologically and radiographically documented locally advanced SCCHN as previously described [18], and had an Eastern Cooperative Oncology 
Group (ECOG) performance status of $0-2$, and adequate organ function to undergo chemotherapy. No prior history of malignancy within the last 5 years (excluding non-melanomatous skin cancer, or in situ cervix cancer) or pre-existing grade 2 or greater peripheral neuropathy was allowed.

\section{Induction chemotherapy and radiation}

The induction regimen has been previously described in detail [18] and is summarized in Fig. 1. Two cycles of induction carboplatin, paclitaxel, and LDFRT were given 21 days apart. All chemotherapy was administered as an outpatient and given as previously reported. Radiation was given on days 1 and 2 of each cycle and consisted of four fractions of $80 \mathrm{cGy}$ each (total dose $640 \mathrm{cGy}$ in 8 fractions), separated by 4 to $6 \mathrm{~h}$. Gross disease (primary tumor and gross nodal disease) was targeted using conformal fields with a maximum $2-\mathrm{cm}$ margin and exclusion of the spinal cord from the induction fields.

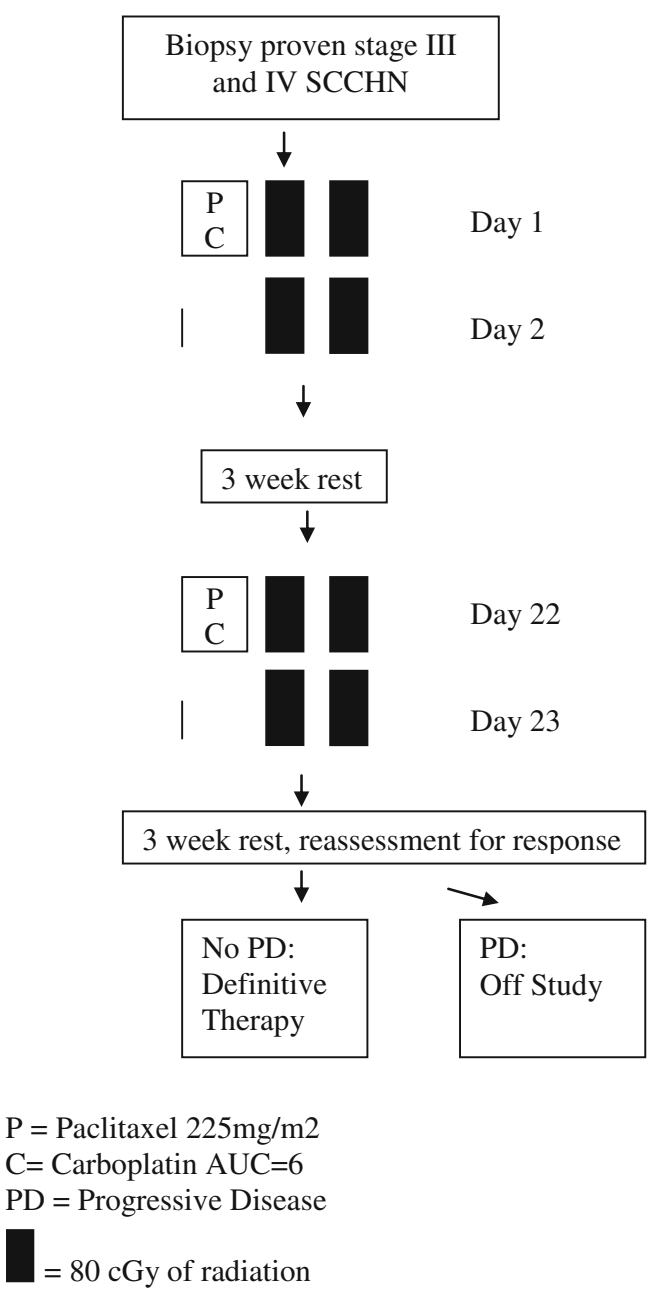

Fig. 1 Induction therapy

\section{Post-therapy evaluation}

Three weeks after the last doses of radiation, patients underwent tumor assessment by pan-endoscopy including primary site assessment by the attending surgeon and radiographic assessment by CT or MRI. Overall response was graded based on the primary and nodal response. Acute toxicity was evaluated using the NCI's Common Toxicity Criteria version 3.0. Late toxicity was determined by review of the patient's medical chart.

Definitive treatment post-induction

Post-induction, the patients were reviewed by a multidisciplinary team including medical oncologists, radiation oncologists, otolaryngologists, radiologists, and pathologists, and postinduction treatment was determined by consensus. Definitive therapy was influenced by response to induction, tolerance of induction, patient wishes, pattern and bulk of residual disease, and medical co-morbidities. As suggested by others [19, 20], patients who had significant responses to induction were treated less intensively with once-daily radiation alone. Patients with bulky residual disease were treated with intensification of therapy with altered fractionation, systemic therapy, or both. All subjects received 3-dimensional planning, and total induction dose of radiation was included into the final dose calculation for definitive therapy. Therefore, the maximum total dose (induction+definitive) for each subject was 7,640 cGy for those receiving once-daily fractionation) or $8,320 \mathrm{cGy}$ for those receiving twice-daily fractionation.

\section{Statistical analysis}

The primary objective of this phase II study was to evaluate antitumor response rates and toxicity of LDFRT and induction chemotherapy in stage III and IVa/b SCCHN as outlined along with design specifications in Arnold et al. [18]. The 5-year rates of progression-free, disease-specific, and overall survival were calculated using the Kaplan-Meier method. Cox proportional hazards models for both overall (OS) and progressionfree survival (PFS) were first considered using a saturated model adjusting for variables including gender, age, primary tumor site, tumor category, nodal category, primary treatment, and concurrent chemotherapy. Multivariate models were developed using backward selection to minimize Akaike's information criteria. All statistics were conducted in R [21].

\section{Results}

Patients

Forty patients with stage III and IVa/b SCCHN were enrolled in this study. The median follow-up for all patients is 83 months 
Table 1 Patient characteristics

\begin{tabular}{|c|c|c|}
\hline Gender & $N$ & Percent \\
\hline Male & 31 & 79.5 \\
\hline Female & 8 & 20.5 \\
\hline \multicolumn{3}{|l|}{ Age (years) } \\
\hline Range & $36-81$ & \\
\hline Median & 54 & \\
\hline \multicolumn{3}{|c|}{ Follow-up time (years) } \\
\hline Range & $0.5-9.9$ & \\
\hline Median & 6.9 & \\
\hline \multicolumn{3}{|l|}{ Primary tumor site } \\
\hline Oropharynx & 15 & 39 \\
\hline Larynx & 13 & 33 \\
\hline Oral cavity & 6 & 15 \\
\hline Hypopharynx & 5 & 13 \\
\hline \multicolumn{3}{|l|}{ Overall stage } \\
\hline III & 9 & 23 \\
\hline IV & 30 & 77 \\
\hline \multicolumn{3}{|l|}{ Nodal stage } \\
\hline N0-N2a & 16 & 41 \\
\hline $\mathrm{N} 2 \mathrm{~b}-\mathrm{N} 3$ & 23 & 59 \\
\hline \multicolumn{3}{|l|}{ Tumor stage } \\
\hline $\mathrm{T} 1, \mathrm{~T} 2$, or $\mathrm{T} 3$ & 21 & 54 \\
\hline $\mathrm{T} 4$ & 18 & 46 \\
\hline \multicolumn{3}{|l|}{ Primary treatment } \\
\hline Chemo+XRT & 20 & 51 \\
\hline XRT alone & 15 & 39 \\
\hline Surgery & 4 & 10 \\
\hline \multicolumn{3}{|l|}{ RT fractionation } \\
\hline Hyperfractionated & 26 & 70 \\
\hline Standard & 10 & 27 \\
\hline Other & 3 & 3 \\
\hline Total & 39 & 100 \\
\hline
\end{tabular}

(range $=7-118$ ). The median follow-up for the 15 survivors is 102 months (range=66-118). Table 1 shows patient demographics. The overall stage was $23 \%$ III, $70 \%$ IVA, and $8 \%$ IVB, and $54 \%$ of patients had T1, T2, or T3 tumors, while $41 \%$ of patients were N0-N2a and $59 \%$ were N2b-N3. All patients were chemotherapy naïve. One patient had a history of previous SCCHN more than 5 years prior to enrollment.

Patients were analyzed on an intent-to-treat basis. Thirtynine patients were evaluable for response, toxicity, local regional control, distant control, and progression-free and overall survival outcomes; one subject was not evaluable because he was lost to follow-up after cycle 1 of induction. Two other patients received one cycle of induction but are included in the outcome analysis; one refused further induction therapy and another progressed in neck nodes. All remaining patients received the full intended dose of induction therapy and only one patient required 1 week delay in administration of cycle 2 secondary to cytopenias.

Acute toxicity and response date

The details of the acute toxicity and response data have been previously reported [18], with only neutropenia (50\%), infection $(8 \%)$, and dermatologic $(8 \%)$, grade 3 and 4 toxicity rates over $5 \%$ and no grade 5 toxicities. The response rates $(\mathrm{CR}+\mathrm{PR})$ was $82 \%$ overall, with only one subject showing progressive disease (PD) in lymph nodes. A primary site RR of $90 \%$ was observed, with no PD at the primary site.

\section{Definitive therapy}

Definitive therapy began 3 weeks the last dose of chemotherapy and LDFRT in all subjects and was determined by multidisciplinary conference consensus. Definitive therapy consisted of: concurrent chemoradiation in 20 patients $(51 \%)$, radiation alone in 15 patients $(39 \%)$, surgery in 2 patients $(5 \%)$, and surgery and radiation in 2 patients $(5 \%)$. Definitive treatment was influenced by response to induction, location of the primary (oral cavity versus other), tolerance of induction, patient wishes, pattern and bulk of residual disease, and medical co-morbidities. Patients who had poor responses to induction were offered surgical therapy or more intensive radiation or chemoradiation. Compliance with definitive therapy was $95 \%$ (38/40 subjects). Radiation was given without treatment interruptions except in two patients. The first patient refused further radiation after 4,260 cGy and underwent salvage surgery, while the second was non-compliant, stopped therapy at 3,880 cGy, and refused further therapy. Five patients underwent adjuvant neck dissections post-radiation with an $80 \%$ pathologic CR rate, and one patient had planned surgery followed by post-operative radiation.

Patients who completed definitive radiation alone received doses ranging from 6,200-7,660 cGy (median 7,200 cGy). Of the 19 patients who received definitive chemoradiation, 16 received hyperfractionated radiation with intra-arterial cisplatin (HYPERRADPLAT) as previously described by Spring et al. in Cancer [22], while three others received concurrent intravenous chemotherapy.

\section{Patient status}

Of the 39 evaluable patients, 15 are still alive without evidence of disease. Fifteen patients have died of SCCHN, and 9 patients have died of other causes. Fifteen patients recurred: 7 distant only, 6 locoregional only, and 2 failed both distantly and locally. First sites of distant failure were the lungs in 7 patients and multiple simultaneous sites in the 
other 2 patients. Absolute rates of locoregional control (LRC) and distant control (DC) are $80 \%$ and $77 \%$, respectively.

Five-year overall survival (OS), disease-specific survival, and progression-free survival (PFS) are $62 \%, 66 \%$, and $58 \%$, respectively. OS and PFS curves are displayed in Fig. 2, which also shows OS stratified by primary tumor site and PFS stratified by T category. On univariate analyses, T category was predictive of PFS $(p<0.001)$, and primary tumor site was predictive of OS $(p<0.001)$. Table 2 displays adjusted hazard ratios for multivariable models of overall and progression-free survival. On multivariate analysis, T4 disease was associated with worse overall survival compared to T1, T2, or T3 disease (HR 5.0; $95 \%$ CI 2.0 13.0). Oral cavity primary tumors as compared to oropharynx tumors were also associated with worse overall survival (HR 17.1; $95 \%$ CI 3.7-78.6) on multivariate analysis. An

A

Overall Survival

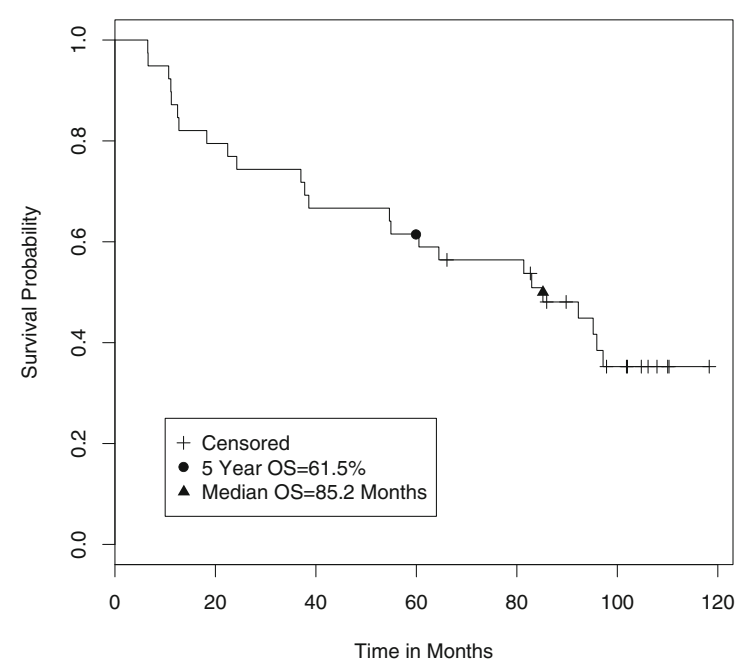

C

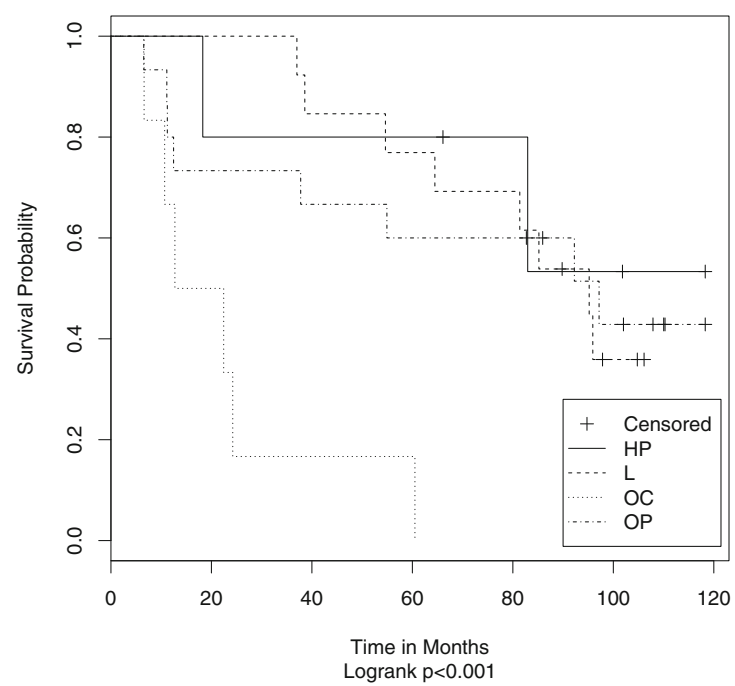

increased risk of recurrence was also associated with those patients with T4 tumors ( $\mathrm{HR}=18.0 ; 95 \% \mathrm{CI} 4.9$ to 65.7$)$ or oral cavity primaries ( $\mathrm{HR}=10.3 ; 95 \% \mathrm{CI} 2.0$ to 52.3$)$.

Late toxicity included 4 pts. with feeding tube dependence greater than 1 year, three cases of laryngeal stenosis, two grade 3 esophageal strictures, one osteoradionecrosis, one upper extremity neuropathy, and one tracheocutaneous fistula postsurgery. Second malignancies occurred in $10(26 \%)$ patients. These included three skin, two head and neck, two lung, one esophageal, one pancreatic, and one rectal cancer.

\section{Discussion}

The initial results of this trial were the first to describe the use of LDFRT as part of a chemotherapy induction regimen

B

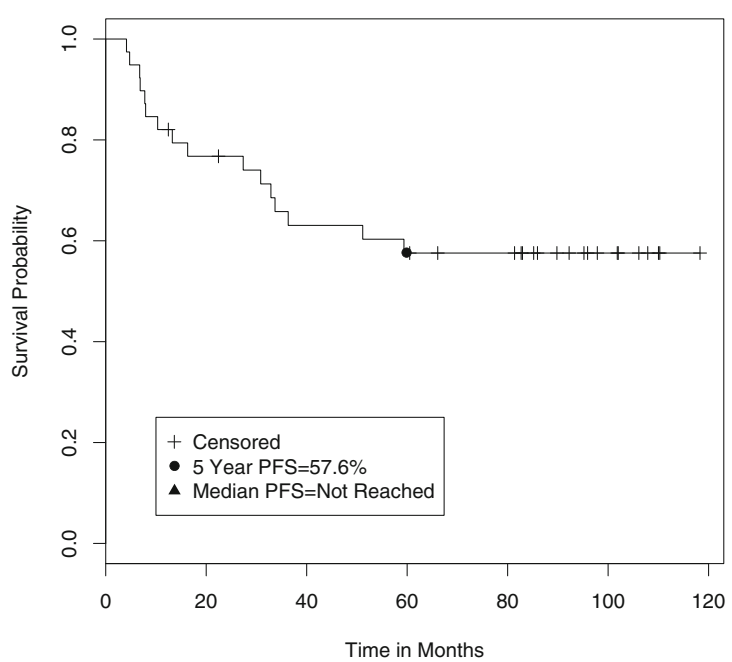

D Progression-Free Survival by T Category

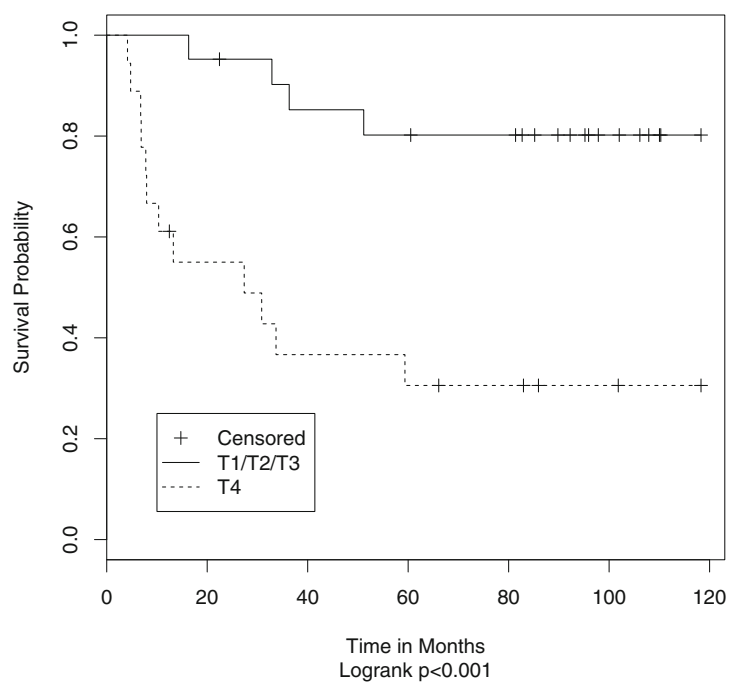

Fig. 2 Kaplan-Meier survival estimates 
Table 2 Adjusted hazard ratios for overall and progression-free survival

${ }^{a}$ Estimates for each covariate have been adjusted for all other covariates listed

Using backwards selection modeling, the best model selected for OS included age, primary tumor site, and t category. The best model selected for PFS included gender, primary tumor site, and $\mathrm{t}$ category. Table 2 presents each of the best models selected and does not adjust for age (in PFS model), gender (in OS model), and $\mathrm{N}$ category, primary treatment, and concurrent chemotherapy in either OS or PFS models as they were not significant and provided poorer overall model fit

\begin{tabular}{llll}
\hline \multirow{2}{*}{ Covariate } & & All patients $N=41$ & \\
\cline { 4 - 4 } & & Hazard ratio $^{\mathrm{a}}(95 \% \mathrm{CI})$ & P value \\
\hline Overall survival & & & 0.09 \\
Age & $>50$ vs. $\leq 50$ years & $2.4(0.9-6.8)$ & 0.18 \\
Primary tumor site & Hypopharynx & $0.3(0.07-1.7)$ & 0.45 \\
& Larynx & $1.5(0.5-4.4)$ & $<0.001$ \\
& Oral cavity & $17.1(3.7-78.6)$ & $<0.001$ \\
Tumor stage & Oropharynx & 1.00 & 0.08 \\
Progression-free survival & T4 vs. T1-T3 & $5.0(2.0-13.0)$ & 0.04 \\
Gender & & & 0.41 \\
Primary tumor site & Male vs. female & $4.0(0.8-18.8)$ & 0.005 \\
& Hypopharynx & $0.1(0.0-1.0)$ & $<0.001$ \\
\hline
\end{tabular}

for SCCHN in human subjects [18]. Using these very low doses of radiation to enhance the effect of chemotherapy is innovative and expands the range of effective doses of radiotherapy. As elucidated by Joiner et al. [23], HRS is the phenomenon by which very low radiation doses actually cause increased cell kill per Gray, compared to higher, more conventional doses. Several groups have shown that HRS is independent of DNA-dependent protein kinase complex used to repair double-stranded DNA damage [16, 24]. HRS does not cause initiation of DNA-repair mechanisms that would otherwise arrest these partially damaged cells at the $\mathrm{G} 2$ phase of the cell cycle, thus favoring pro-apoptotic pathways [24]. In other words, at the cellular level, without the double-strand breaks caused by standard dose radiation as a trigger, the cell does not recognize the DNA damage from LDRT as significant, does not initiate DNA repair, and the cell dies an apoptotic death because of a lack of recognition of LDRT's cellular damage.

Through the successful addition of LDFRT to induction chemotherapy, we have demonstrated that this novel strategy is tolerable and provides an excellent response rate $(82 \%$ overall and $90 \%$ at the primary site). The updated 5-year results presented in this manuscript demonstrate favorable long-term survival outcomes and no unexpected long-term complications when using this unique approach. This would argue against any negative impact from accelerated repopulation when using induction LDFRT. LRC of $80 \%$ and 5 year OS of $62 \%$ compare quite favorably with historical controls $[4-6,25,26]$ for treating locally advanced SCCHN. Oral cavity cancers and $\mathrm{T} 4$ primary tumors were the factors in our series associated with worse PFS and OS on multivariate analysis.

Table 3 Comparison of UK H\&N 11 regimen to TPF containing induction regimens for locally advanced H\&N cancer

\begin{tabular}{|c|c|c|c|c|c|c|c|c|}
\hline Trial & $\begin{array}{l}\text { Induction } \\
\text { regimen }\end{array}$ & $\begin{array}{l}\text { Definitive } \\
\text { treatment }\end{array}$ & $\begin{array}{l}\text { Percent } \\
\text { stage IV }\end{array}$ & $\begin{array}{l}\text { Grade } 3,4 \\
\text { neutropenia }\end{array}$ & $\mathrm{RR}$ & LRC & $\begin{array}{l}\text { Metastatic } \\
\text { rate }\end{array}$ & OS \\
\hline TAX $323^{11}(n=177)$ & TPF arm & Radiation & NR & $77 \%$ & $68 \%$ & $52 \%$ & $13 \%$ & $37 \%$ (3 years) \\
\hline TAX $324^{12,13}(n=255)$ & TPF arm & Chemo-radiation & $82.5 \%$ & $83 \%$ & $72 \%$ & $70 \%$ & $5 \%$ & $52 \%(5$ years $)$ \\
\hline UK H\&N $11(n=39)$ & $\mathrm{C}, \mathrm{P}, \mathrm{LDFRT}$ & Radiation, chemoradiation, surgery & $77.0 \%$ & $50 \%$ & $82 \%$ & $80 \%$ & $23 \%$ & $62 \%(5$ years $)$ \\
\hline
\end{tabular}

$\mathrm{TPF}=$ docetaxel, cisplatin, and fluorouracil

$C$ Carboplatin

$P$ Paclitaxel

$L D F R T$ low-dose fractionated radiation

$R R$ response rate

$O S$ overall survival

$L R C$ local regional control

$N R$ not reported 
LDFRT, carboplatin, and paclitaxel had similar toxicities to induction carboplatin and paclitaxel in SCCHN patients as reported by others $[27,28]$. LDFRT did not increase the rate of radiation-induced grade 3 or 4 toxicities. In addition, definitive therapy was not delayed or interrupted. When compared to other published trials of induction paclitaxel and carboplatin, our data demonstrates favorable primary site and overall RRs using LDFRT with carboplatin and paclitaxel [18, 27-30].

Our study has limitations in terms of the heterogeneity of post-induction treatment given. While definitive therapy did differ, it was based on stratification by response to induction therapy, an accepted practice advanced by others $[19,20]$. The strategy of utilizing response to induction as a guide to the intensity of subsequent therapy did not lead to worsening survival; however, conclusions regarding this approach remain exploratory in nature, because of the small sample size and heterogeneity of the population. We are now able to report the additional endpoint of five-year survival analysis and long-term rates of locoregional and distant control, which are comparable to historic controls. This unique regimen of LDFRT with carboplatin and paclitaxel as an induction does show excellent long-term outcomes, without detriment to survival, or compliance with definitive postinduction therapy, and warrants further investigation.

Since the design and completion of this trial, the status of induction chemotherapy for head and neck cancer has evolved. The results of the TAX 323 and TAX 324 trials have led to docetaxel, cisplatin, and fluorouracil (TPF) being adopted by many as the induction regimen of choice [11-13]. In some patients with SCCHN, this regimen has been too toxic to deliver with a reasonable expectation of compliance and tolerance without treatment delays. Table 3 is a comparison of the response rates, acute toxicity, LRC, distant metastases, and overall survival of patients treated on TAX 323, TAX 324, and the currently presented UK H\&N 11 trial. The rates of acute grade 3 or 4 neutropenia were $77 \%$ and $83 \%$ in the TPF arms of the TAX trials, whereas it was only $50 \%$ in our trial. Additionally, the overall response rates to TPF in the TAX trials are similar to our trial: $68 \%$ and $72 \%$ in the TAX trials versus $82 \%$ in our present study. While our study was only a non-randomized phase II trial, the LRC rate is comparable in our study: $80 \%$ in UK H\&N 11 versus the TPF arms of TAX 323 (52\%) and TAX $324(70 \%)$. It is possible that the addition of LDFRT to the induction regimen contributed to this improved LRC. In contrast to the LRC rates, the distant metastatic rate is higher in our trial $(23 \%)$ when compared to the TPF arms of the TAX trials (5\% in TAX 324 and $13 \%$ in TAX 323). This may be partly explained by the fact that a large proportion of our subjects $(77 \%)$ had stage $\mathrm{IVa} / \mathrm{b}$ disease, and $46 \%$ of subjects had T4 tumors. This inherently leads to a higher risk of metastatic disease. In addition, our study only included two cycles of induction therapy, compared to others which used three cycles, which could have impacted the distant metastatic rate in our study.

\section{Conclusions}

Our unique induction regimen of low-dose fractionated radiation, carboplatin, and paclitaxel has shown excellent rates of initial overall response ( $82 \%), 5$ year LRC ( $80 \%)$, and 5 year overall survival $(62 \%)$. The acute toxicity profile of this regimen is markedly less than that seen with three-drug induction regimens such as TPF, which have high rates of grade 3 or 4 neutropenia. This may reduce potential delays and alterations in definitive therapy. This long-term follow-up study demonstrates no evidence of diminished therapeutic efficacy with standard platinum-based definitive concurrent chemoradiation following this induction regimen. These results warrant further investigation of LDFRT as part of a systemic induction regimen. That is the focus of investigation for a planned randomized study comparing induction chemotherapy with or without the addition of LDFRT, using the regimen from $\mathrm{H} \& \mathrm{~N} 11$ as the experimental arm. Because we have demonstrated the tolerability of concurrent chemoradiation following this induction regimen, the induction treatment in the randomized trial will be followed by standard platinumbased concurrent chemoradiation in all patients.

In summary, the long-term results using LDFRT in combination with carboplatin and paclitaxel as induction in SCCHN indicate its safety and efficacy with promising overall survival, an excellent rate of locoregional control, and an acceptable distant metastatic rate.

Conflict of interest statement Bristol-Myers Squibb provided research funds to the University of Kentucky to support this research. Aside from these research funds, provided solely to perform the research in this study, the authors declare that they have no conflict of interest.

\section{References}

1. Siegel R, Ward E, Brawley O et al (2011) Cancer Statistics, 2011the impact of eliminating socioeconomic and racial disparities on premature cancer deaths. CA Cancer J Clin 61:212-236

2. Seiwert TY, Cohen EEW (2005) State-of-the-art management of locally advanced head and neck cancer. Br J Cancer 92:1341-1348

3. Denis F, Garaud P, Bardet E et al (2004) Final results of the 94-01 French Head and Neck Oncology and Radiotherapy Group randomized trial comparing radiotherapy alone with concomitant radiochemotherapy in advanced-stage oropharynx carcinoma. J Clin Oncol 22:69-76

4. Adelstein DJ, Li Y, Adams GL et al (2003) An intergroup phase III comparison of standard radiation therapy and two schedules of 
concurrent chemoradiotherapy in patients with unresectable squamous cell head and neck cancer. J Clin Oncol 21:92-98

5. Forastiere AA, Goepfert H, Maor M et al (2003) Concurrent chemotherapy and radiotherapy for organ preservation in advanced laryngeal cancer. N Eng J Med 349:2091-2098

6. Forastiere A, Maor M, Weber R, et al. (2006) Long-term results of intergroup RTOG 91-11: a phase III trial to preserve the larynxinduction cisplatin/5FU and radiation therapy versus concurrent cisplatin and radiation therapy versus radiation therapy, ASCO National Proceedings

7. Bernier J, Domenge C, Ozsahin M et al (2004) Postoperative irradiation with or without concomitant chemotherapy for locally advanced head and neck cancer. N Engl J Med 350:1945-1952

8. Cooper JS, Pajak TF, Forastiere AA et al (2004) Postoperative concurrent radiotherapy and chemotherapy for high-risk squamous cell carcinoma of the head and neck. N Engl J Med 350:19371944

9. Bernier J, Cooper JS, Pajak TF et al (2005) Defining risk levels in locally advanced head and neck cancers: comparative analysis of concurrent postoperative radiation plus chemotherapy of the EORTC (\#22931) and RTOG (\#9501). Head Neck 27:843-850

10. Pignon JP, le Maitre A, Maillard E et al (2009) Meta-analysis of chemotherapy in head and neck cancer (MACH-NC): an update on 93 randomised trials and 17,346 patients. Radiother Oncol 92:4-14

11. Vermorken JB, Remenar E, van Herpen C et al (2007) Cisplatin, fluorouracil, and docetaxel in unresectable head and neck cancer. N Engl J Med 357:1695-1704

12. Posner MR, Hershock DM, Blajman CR et al (2007) Cisplatin and fluorouracil alone or with docetaxel in head and neck cancer. $\mathrm{N}$ Engl J Med 357:1705-1715

13. Lorch JH, Goloubeva O, Haddad RI et al (2011) Induction chemotherapy with cisplatin and fluorouracil alone or in combination with docetaxel in locally advanced squamous-cell cancer of the head and neck: long-term results of the TAX 324 randomised phase 3 trial. Lancet Oncol 12:153-159

14. Joiner MC, Marples B, Lambin P et al (2001) Low-dose hypersensitivity: current status and possible mechanisms. Int J Radiat Oncol Biol Phys 49:379-389

15. Marples B, Cann NE, Mitchell CR et al (2002) Evidence for the involvement of DNA-dependent protein kinase in the phenomena of low dose hyper-radiosensitivity and increased radioresistance. Int J Radiat Biol 78:1139-1147

16. Dey S, Spring P, Arnold SM et al (2003) Low dose fractionated radiation potentiates the effects of Paclitaxel in wild-type and mutant p53 head and neck tumor cell lines. Clin Cancer Res 9:1557-1565

17. Spring PM, Arnold SM, Shajahan S et al (2004) Low dose fractionated radiation potentiates the effects of taxotere in nude mice xenografts of squamous cell carcinoma of head and neck. Cell Cycle 3(4):479-485
18. Arnold SM, Regine WF, Ahmed MM et al (2004) Low-dose fractionated radiation as a chemopotentiator of neoadjuvant paclitaxel and carboplatin for locally advanced squamous cell carcinoma of the head and neck: results of a new treatment paradigm. Int J Radiat Oncol Biol Phys 58:1411-1417

19. Worden FP, Moyer J, Lee JS et al (2009) Chemoselection as a strategy for organ preservation in patients with T4 laryngeal squamous cell carcinoma with cartilage invasion. Laryngoscope 119 (8):1510-1517

20. Worden FP, Kumar B, Lee JS et al (2008) Head and neck cancer: chemoselection as a strategy for organ preservation in advanced oropharynx cancer: response and survival positively associated with HPV16 copy number. J Clin Oncol 26(19):3138-3146

21. R Development Core Team (2010) R: a language and environment for statistical computing. R Foundation for Statistical Computing, Vienna, Austria, ISBN 3-900051-07-0, URL http://www.R-project.org

22. Spring PM, Valentino J, Arnold SM et al (2005) Long-term results of hyperfractionated radiation and high-dose intraarterial cisplatin for unresectable oropharyngeal carcinoma. Cancer 104(8):1765-1771

23. Short SC, Kelly J, Mayes CR, Woodcock M, Joiner MC (2001) Low-dose hypersensitivity after fractionated low-dose irradiation in vitro. Int J Radiat Biol 77(6):655-664

24. Martin L, Marples B, Coffey M, Lawler M, Hollywood D, Marignol L (2009) Recognition of O6MeG lesions by MGMT and mismatch repair proficiency may be a prerequisite for lowdose radiation hypersensitivity. Radiat Res 172(4):405-413

25. Ang K, Zhang RH, Wheeler DI et al (2010) Phase III trial (RTOG 0129) of two radiation-cisplatin regimens for head and neck carcinomas (HNC): impact of radiation and cisplatin intensity on outcome. J Clin Oncol 28(15 suppl):5507

26. Bonner JA, Harari PM, Giralt J et al (2006) Radiotherapy plus Cetuximab for squamous cell carcinoma of the head and neck. N Engl J Med 354:567-578

27. Dunphy FR, Dunleavy TL, Harrison BR et al (2001) Induction paclitaxel and carboplatin for patients with head and neck carcinoma. Analysis of 62 patients treated between 1994 and 1999. Cancer 91:940-948

28. Machtay MM, Rosenthal DI, Hershock D et al (2002) Organ preservation therapy using induction plus concurrent chemoradiation for advanced resectable oropharyngeal carcinoma: A University of Pennsylvania Phase II trial. J Clin Oncol 20:3964-3971

29. Vokes E, Stenson K, Rosen F et al (2003) Weekly carboplatin and paclitaxel followed by concomitant paclitaxel, fluorouracil and hydroxyurea chemoradiotherapy: curative and organ-preserving therapy for advanced head and neck cancer. J Clin Oncol 21:320-326

30. Bouillet T, Morere JF, Depreaux G et al (1999) Phase II study of paclitaxel (P) twice a week as a radiosensitizer, after paclitaxelcarboplatin $(\mathrm{C})$ induction in stage III-IV head and neck carcinoma. Proc Am Soc Clin Oncol 18:403a, (abstr) 